\title{
Trayectorias de vida e infarto agudo de miocardio: vivencias de varones de la ciudad de Bogotá
}

\author{
Lifelines and acute myocardial infarction: \\ men experiences in Bogotá city
}

Lorena Mesa Melgarejo ${ }^{1,2}$

Forma de citar: Mesa L. Trayectorias de vida e infarto agudo de miocardio: vivencias de varonas de la ciudad de Bogotá. Rev Univ Ind Santander Salud. 2016; 48(3): 375-383. DOI: http://dx.doi.org/10.18273/revsal.v48n3-2016011 @ (1)

\section{RESUMEN}

Introducción: Los riesgos de las enfermedades cardiovasculares comienzan desde la vida fetal y continúan a través de la infancia, la adolescencia y la vida adulta, aproximarse a su trayectoria de construcción resulta fundamental para la atención en salud. Objetivo: Conocer y comprender las trayectorias que estructuran los estilos de vida de varones que han sufrido infarto agudo de miocardio. Metodología: Estudio de carácter cualitativo descriptivo. Los hombres invitados a participar se seleccionaron a partir de la base de datos de ingreso y egreso de pacientes de la unidad de cuidados coronarios del Hospital San José de Bogotá. Se logra una saturación de categorías hacía la entrevista número 11. Los datos fueron analizados siguiendo la propuesta de Janice M. Morse. Resultados: A partir de los datos analizados se construyen dos categorías: 1. Trabajo, masculinidad y socialización: marco de los estilos de vida y 2. "Mi pequeño modo de entender": Autocomprensión del fenómeno. En conjunto estas categorías permiten comprender cómo la reproducción social y el mundo del trabajo de los varones ordenan las opciones y decisiones sobre los estilos de vida que ellos asumen. Conclusión: La aproximación comprensiva a las trayectorias que estructuran los estilos de vida de varones que han sufrido infarto agudo de miocardio desde sus voces y representaciones nos muestran cómo el mundo del trabajo masculino sobredetermina muchos de los comportamientos y elecciones de la cotidianidad (nocivos), como una dialéctica entre agencia y estructura.

Palabras Clave: Infarto del miocardio, investigación cualitativa, estilo de vida, acontecimientos que cambian la vida, masculinidad.

\begin{abstract}
Introduction: The risk for cardiovascular diseases starts with fetal life and the accumulation continues throughout the whole life; the approximation to how those risks are constructed is a key point for health care. Objetive: To understand the lifelines of men in Bogota who suffered acute myocardial infarction and how those structured his

1. Universidad Nacional de Colombia. Bogotá, Colombia

2. Fundación Universitaria de Ciencias de la Salud. Bogotá, Colombia

Correspondencia: Lorena Mesa Melgarejo. Dirección: Carrera 33 No. 25F-10. Torre 7 - Ap. 2015. Bogotá Colombia. Correo electrónico: lpmesam@unal.edu.co. Teléfono: +71 3929455.
\end{abstract}

Recibido: 18/01/2016

Aprobado: 09/03/2016

Publicado online: 24/06/2016 
lifestyle. Methodology: Qualitative descriptive study. The participants were men hospitalized in the Coronary care unit of the Hospital de San José (Bogota, Colombia). The saturation was possible at 11th interview. For the data analysis was used the Janice M. Morse proposal. Results: Two categories were synthesized: 1. Work, masculinity and socialization: lifestyle framework, and 2. "My little way for understanding": Self- comprehension of the phenomenon. Conclusion: The qualitative approximation to the men's lifelines and how those have been structured, show us the overdetermination of the work and masculinity for developing harmful behaviors and choices during the daily life.

Keywords: myocardial infarction; Qualitative research; Life styles; Life Change Events; Masculinity.

\section{INTRODUCCIÓN}

Las enfermedades cardiovasculares son la principal causa de muerte en todo el mundo. De acuerdo con los datos de la Organización Mundial de la Salud -OMSse calcula que en el 2012 murieron por esta causa 17.5 millones de personas, lo cual representa un 31\% de todas las muertes registradas en el mundo, de este porcentaje, 7.4 millones se debieron a infarto agudo de miocardio (IAM) ${ }^{1}$. En Colombia para el 2011 (último año reportado oficialmente) el $23,5 \%$ del total de muertes fueron causadas por una patología de origen cardiovascular y el $56,3 \%$ de ese porcentaje, fue por enfermedad isquémica cardiaca ${ }^{2}$. Según el reporte del Instituto Nacional de Salud -INS-, entre 1998 y 2011 se observó mayor mortalidad masculina con un porcentaje del $51.4 \%$, de modo que, como afirma el Ministerio de Salud: "el infarto agudo de miocardio fue responsable de la muerte de 16.000 hombres de las 29.000 personas que fallecieron en 2011 por esa causa, lo cual indica que fallecieron, por esta enfermedad, 43 varones por día"3.

Desde los años 30 y 40 del siglo pasado se comenzó a reconocer que la hipertensión, el colesterol, la malnutrición, la obesidad, el cigarrillo, la inactividad física y el estrés psicosocial como factores que contribuyen significativamente a la enfermedad cardíaca. En las últimas 4 décadas se ha acumulado suficiente evidencia que demuestra que estos factores de riesgo cardiovascular (FRCV) reducen la expectativa de vida entre 10 y 15 años $^{4}$. Estos FRCV, que se materializan en las personas, a su vez se encuentran afectados por el inadecuado acceso a los sistemas de salud, la mala calidad de los proveedores de asistencia sanitaria, la reducción de la financiación de la asistencia sanitaria, la falta de infraestructuras adecuadas y problemas de acceso a una dieta adecuada ${ }^{5}$, es decir, están relacionados con aspectos más allá de los individuos, están en estrecha relación con el mundo social y con los modos en que las personas pueden tomar decisiones sobre su salud dependiendo de las posibilidades que el contexto les ofrece.
Los estudios sobre FRCV y su relación con las trayectorias de vida de las personas con enfermedades crónicas demuestran que las enfermedades cardiovasculares son el resultado de la acumulación de riesgos a través de la vida de las personas. Estos estudios además reconocen que los riesgos deben ser reducidos y prevenidos a lo largo de la vida ${ }^{6,7}$. De acuerdo con este principio, los riesgos de enfermedad cardiovascular comienzan desde la vida fetal y continúan a través de la infancia, la adolescencia y la vida adulta ${ }^{8}$.

Por otro lado y desde la epidemiologia social, diversos estudios revelan la existencia de una fuerte asociación entre la posición socioeconómica y la enfermedad cardiovascular $^{9-12}$, es decir, una situación económica desfavorable, un bajo nivel de estudios, una ocupación de bajo nivel y una mala situación en el mercado laboral, se consideran FRCV asociados; sin embargo no es claro a través de qué mecanismos se produce esta asociación, requiriendo mayor investigación en este campo $^{13}$. Respecto al tema, investigadores de la línea de enfermedades crónicas del Instituto Nacional de Salud de Helsinki (Finlandia), consideran que se requiere: 1). Desarrollar estudios con una perspectiva basada en la evolución de toda la vida, 2). Desarrollar marcos de referencia conceptuales e indicadores para otros mecanismos explicativos alternativos, 3) Desarrollo de nuevos diseños y métodos de estudio, entre otros ${ }^{13}$.

En línea con lo anterior, el estudio de Emsile concluye que son relativamente escasos los estudios que exploran la experiencia de las personas con enfermedades cardiovasculares desde la perspectiva de género y que comparen las experiencias entre hombres y mujeres, pero además, más escasos aún son los estudios que tienen en cuenta la influencia de las masculinidades sobre las creencias y comportamientos relacionados con este tipo de enfermedades ${ }^{14}$.

Surge entonces la necesidad de aproximarse de manera comprensiva al análisis de los FRCV y a los estilos de vida para generar acciones distintas y posiblemente 
más significativas y contextualizadas. Por lo anterior el objetivo de este estudio fue conocer y comprender las trayectorias que estructuran los estilos de vida de varones que han presentado infarto agudo de miocardio. Vale la pena mencionar que el concepto de trayectoria, desde la perspectiva sociológica, hace referencia a la construcción del "universo existencial de los individuos a través de la organización de sus secuencias temporales de vida"15 existencia que se construye a partir de relaciones objetivas; para Bourdieu citado por Lera y cols, el estudio de las trayectorias posibilitan una "mirada integral de las posiciones objetivas transitadas y ocupadas por los actores, teniendo en cuenta la estructura y volumen de los diferentes capitales disponibles, no como enumeración de acontecimientos" $"$.

\section{METODOLOGÍA}

Para aproximarse comprensivamente a la trayectoria de vida de hombres que han sufrido IAM se desarrolló un estudio de carácter cualitativo descriptivo. Siguiendo a Vasilachis, en términos generales el interés de la perspectiva cualitativa está en conocer la manera como el mundo de la vida es comprendido, experimentado y/o producido, para lo cual se emplean métodos de análisis y de explicación flexibles y sensibles tanto a las particularidades de las personas como de los contextos sociales en los que los datos (sentidos, significados, constructos) son producidos ${ }^{17}$.

Selección de participantes: Las personas invitadas a participar se seleccionaron a partir de la base de datos de ingreso y egreso de pacientes de la unidad de cuidados coronarios del Hospital San José de Bogotá, quienes habían estado hospitalizados en el periodo de enero del 2013 a junio del 2014 (entre dos y un año del evento), los invitados fueron solamente varones, debido al objetivo propuesto para el estudio. Con este listado se hizo un primer contacto vía telefónica, las personas interesadas en participar fueron convocadas para realizar la entrevista en una oficina privada del Hospital destinada para tal fin o en su lugar de residencia, según la decisión de los participantes. Las entrevistas fueron realizadas por la investigadora principal.

Siguiendo la lógica del muestreo teórico, el cual tuvo como principal referente la información nueva reportada por los entrevistados ${ }^{18}$, se logra una saturación de los temas emergentes con la entrevista número 11, momento en que se dejó de contactar más personas.

Recolección y análisis de datos: La recolección de datos se hizo por medio de entrevistas semiestructuradas, siguiendo un guion temático construido a partir de la literatura sobre estilos de vida en relación con la trayectoria de vida. Las entrevistas tuvieron una duración entre 58 y 72 minutos. Posteriormente eran transcritas literalmente, con el objetivo de conservar toda la riqueza del discurso de los participantes. Los datos fueron analizados siguiendo la propuesta de Janice M. Morse conocida como el proceso de codificación. Este proceso hace referencia a un microanálisis de los relatos de cada participante revisando línea a línea las entrevistas para generar hallazgos relacionales y con niveles de abstracción ${ }^{19}$. Luego se compararon los códigos extraídos de las diversas entrevistas realizadas para interpretarlos y relacionarlos en términos de categorías emergentes. El proceso de análisis de información se apoyó en el software AtlasTi.

Reflexividad: La reflexividad es entendida como un proceso transversal y fundamental del rigor en la investigación cualitativa, en el cual la persona que investiga hace conciencia de sus posicionamientos de partida y de su estructura teórico metodológica a la hora de interpretar la experiencia verbalizada por el otro y del "yo contextualizado" a medida que el proceso de investigación avanza ${ }^{20}$. Teniendo en cuenta estos elementos, es necesario explicitar que, los posicionamientos de partida se pueden resumir en:

1. El reconocimiento de la subjetividad y la intersubjetividad como posibilidad de generación de conocimiento. Se considera una existencia de correlatos para la interpretación de la realidad, no de un único metarrelato que todo lo explica, ni una única verdad sobre lo que es y no es la realidad.

2. La comprensión de la salud como proceso social, inmerso en las relaciones sociales, cuyos significados se incorporan y construyen en los sujetos a partir del tejido de socialización.

3. Lenguaje como ordenador de la realidad, por ello la importancia de la comunicación y de la verbalización para aproximarse a las interpretaciones del mundo del otro, quien es histórico, dinámico, cambiante y sujeto a las condiciones de su contexto.

Consideraciones éticas: El proyecto fue aprobado por el Comité de Investigación de la Facultad de Enfermería de la Fundación Universitaria de Ciencias de la Salud y el Comité de Ética de Investigaciones en Seres Humanos del Hospital de San José. Para la realización de las entrevistas se solicitó a los participantes la firma de un consentimiento informado, en el cual se dejaba por escrito el objetivo del estudio, la justificación y el compromiso de confidencialidad, respeto y anonimato. A cada participante se le entrego una copia firmada del consentimiento. 
La información transcrita y los textos que surgieron de las grabaciones fueron conocidos por la investigadora y la persona encargada de transcribir la información, quien firmó un documento de confidencialidad de los datos. Los archivos correspondientes quedaron bajo la custodia del coordinador del proyecto y la información obtenida solo fue utilizada para los fines de esta investigación.

\section{RESULTADOS}

Los 11 participantes tenían edades comprendidas entre los 55 y 90 años; la mayoría de ellos nacieron en la ciudad de Bogotá, también refirieron municipios como: Barbosa, Prado, Cúcuta, Guateque, Manizales y Cáqueza, sin embargo al momento de la entrevista, todos vivían en Bogotá. Igualmente, la mayoría son casados y desempeñaron labores de agricultura, mecánica, vigilancia privada, administración, carpintería, entre otras (Tabla 1). Hombres cuyos relatos de vida reflejan provenir de una clase media (obrera) quienes empezaron a trabajar desde edades tempranas, o por lo menos desde edades que en nuestro contexto no son socialmente esperadas; quienes asumieron el rol de proveedores de sus familias extensas o de autosostenimiento, para luego ser proveedores de sus familias nucleares.

Tabla 1. Características sociodemográficas de los participantes.

\begin{tabular}{ccccc}
\hline PARTICIPANTE & EDAD & PROCEDENCIA & ESTADO CIVIL & OCUPACIÓN \\
\hline 1 & 55 & Bogotá & Separado & Vigilante (Parqueadero) \\
2 & 76 & Barbosa (Santander) & Casado & Independiente (taller de mecánica) \\
3 & 61 & Bogotá & Casado & Mecánico \\
4 & 65 & Bogotá & Casado & Comandante (Bomberos) \\
5 & 76 & Prado (Tolima) & Casado & Agricultor \\
6 & 63 & Cunday (Tolima) & Casado & Independiente (Comercio) \\
7 & 69 & Cáqueza(Cundinamarca) & Soltero & Administrador Público \\
8 & 90 & Manizales (Caldas) & Casado & Ebanista \\
9 & 86 & Guateque (Boyacá) & Casado & Conductor \\
10 & 75 & Bogotá & Casado & Mecánico \\
11 & 58 & Cúcuta (Norte de Santander) & Unión libre & Abogado \\
\hline
\end{tabular}

“yo soy el mayor de mis hermanos entonces a mí me toco asumir a los 15 años el rol de padre y ponerme a trabajar, por eso yo trabaje tan temprano, porque primero me toco hacer el reemplazo de él \{padre\}” 7-8:22

" $\{$ mi vida fue\} muy dura, yo salí muy pequeño muy joven de la casa" 9-7:44

"yo me Sali de la casa a los 7 años, me volé del lado de mis padres, yo no volví a la casa sino me rebuscaba por ahi la comida, lavar buses, barrer buses, después de eso ya fui conocido y me metí de ayudante en los buses" 10-7:45

Respecto a la escolaridad, se encontró diversidad de niveles, algunos habían hecho hasta el nivel de primaria, otros secundaria y nivel técnico, solo un participante comentó tener nivel de posgrado. Estas diferencias de nivel educativo no resultaron ser realmente determinantes a la hora de la toma de decisiones sobre la salud de los hombres entrevistados como se presentará más adelante, pues tiene un peso mayor el mundo laboral.

\section{¿Cómo se interpretaron sus trayectorias de vida?}

A partir de los datos analizados se construyen dos categorías emergentes: 1. Trabajo, masculinidad y socialización: marco de los estilos de vida y 2. "Mi pequeño modo de entender": Autocomprensión del fenómeno. En conjunto estas categorías permiten comprender cómo la reproducción social y el mundo del trabajo de los varones ordenan las opciones y decisiones sobre los estilos de vida que ellos asumen (Figura 1). A partir de éstas se puede entender cómo se van erigiendo las trayectorias de vida de los varones entrevistados y la manera como estas, a su vez, otorgan los marcos de comprensión sobre el infarto, la noción de riesgo y de estilo de vida.

1. Trabajo, masculinidad y socialización: marco de los estilos de vida: Al indagar sobre la experiencia de vida desde lo cotidiano, los relatos de los hombres reflejan una transversalidad del papel del trabajo en 
la construcción de hábitos y sentidos sobre la salud, la alimentación, la actividad física $\mathrm{y}$, en general sobre lo que conocemos como estilos de vida. Es alrededor de la labor que han desempeñado los participantes que se generan prácticas como el consumo de cigarrillo o alcohol, pues en los espacios después de la jornada laboral, estas prácticas se entienden como "naturales" o como extensiones de su labor, pero en relación con el ocio.

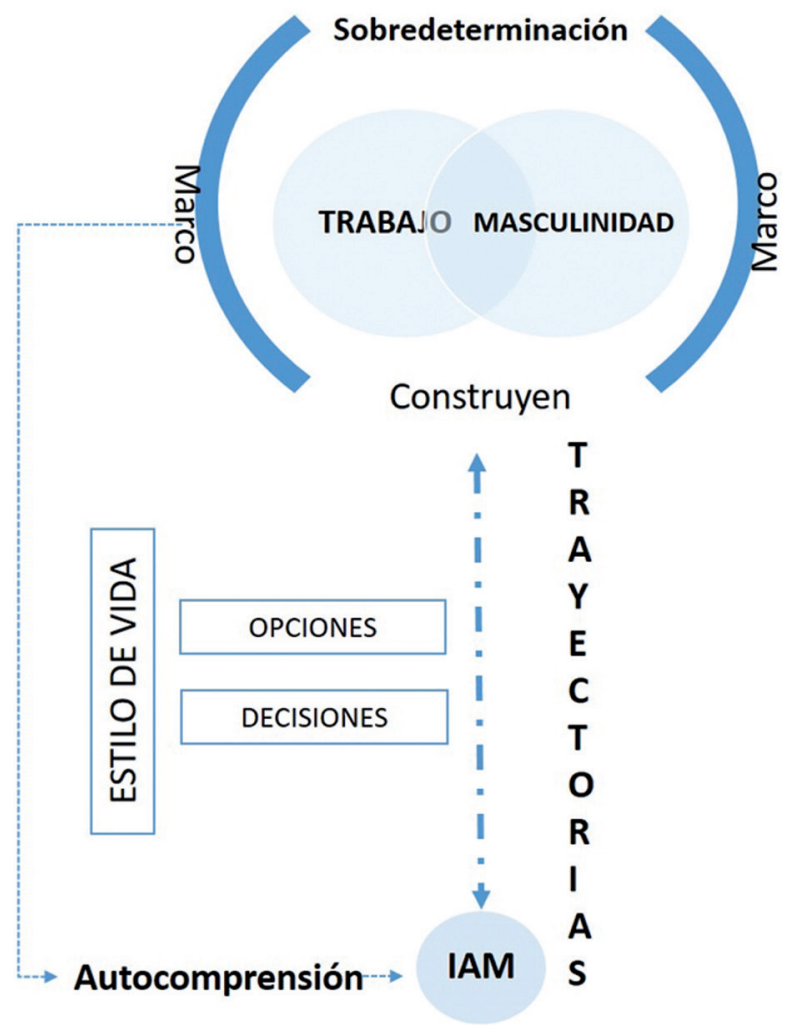

Figura 1. Diagrama de categorías emergentes y relaciones.

"de los compañeros se aprende a fumar, a tomar, con los amigos y pues también estuve en el ejército entonces allá también, dejaba dos o tres meses y volvía a fumar"1-5:24 "bueno en mi época de joven nosotros fumamos hasta tabaco, eso lo normal de uno varón" $8-5: 26$

"en ese trabajo se toma mucho, se fuma mucho"4-4:18

Situación similar ocurre con las decisiones y opciones sobre la alimentación, pues ellos refieren que comen lo que los lugares cercanos a sus trabajos les pueden ofertar. Por otro lado, esta alimentación en el contexto de la región andina suele ser alta en carbohidratos.

"Yo duré 30 años manejando buses de aqui a pasto, Ipiales... en el bus uno aguantaba más hambre que ratón de iglesia, porque uno cogía una ruta y los comederos (restaurantes) quedaban muy distantes... La comida que daban en los restaurantes: sopa, gallina a toda hora, y de harinas hasta el borde del plato" 9-10:5, 33, 38, 43.

"la alimentación era mucha harina, empecé a trabajar como a los 12 años y ahi es cuando uno come en la calle y esa comida es lo que lo degenera mucho" 1-17:155

Los varones mayores aprendieron además a familiarizarse con las bebidas alcohólicas desde temprana edad, pero bebidas que desde lo cultural no se reconocen como "alcohólicas" por el contrario se relacionan con un alimento importante para el trabajo y el esparcimiento de los hombres:

"a mí no me gusta el licor, yo fui criado fue con guarapo, es mejor y más dulce, tiene más calcio y da fuerza para el trabajo 10-14:110

"la chicha es más alimento todavía, porque eso es maiz11:15-114

Llama la atención que el consumo cotidiano de alimentos fritos durante los momentos de descanso en el trabajo no se refiere como hábito alimenticio. Se mencionó varias veces la empanada, producto que suele consumirse en las horas de la mañana. Este tipo de alimentos de alto contenido graso no se reconocen como una fuente ni de riesgo ni como parte de un patrón o hábito alimentario, a pesar de que se consume frecuentemente, incluso algunos entrevistados, refieren su consumo diario.

"empanadita bastante, pero eso solo para las onces \{merienda\}"7-9:134

"sí, desgraciadamente uno comía mucha grasa y no se cuidaba porque pues era lo normal" 2-13:98

"Chicharroncito, chorizo, la fritanga eso era de dos, tres [veces] por semana” 3-10:14

Al indagar por el ejercicio físico, se hace referencia a jugar futbol ocasionalmente con los compañeros de trabajo e irse en bicicleta o caminando del trabajo a la casa y viceversa como actividad física; los varones consideran que la extensa jornada laborar y las cargas en el trabajo son per se actividad física.

En las trayectorias de vida de los varones entrevistados, el espacio de lo laboral resulta determinante para la construcción, aprendizaje e incorporación de los estilos de vida, es muy poca la relación que la familia tiene en este proceso de construcción desde los textos de los entrevistados, lo cual puede estar vinculado a la salida a temprana edad de sus hogares y familias de origen. La familia emerge para referirse a dolencias que se heredaron y que se relacionan con el IAM. 
"en la familia si hay algunos que han sufrido de diabetes e hipertensión, inclusive han muerto de diabetes" 6-7:12

"mi mamá murió de un paro respiratorio, pero es que ella fumaba" 6-7:13

"sé que mi abuelo si fue diabético, que una tía murió de diabetes" 7-8:11

Otra dimensión que se relaciona con lo laboral como estructurantes de los estilos de vida es la idea de masculinidad que subyace en las historias de los entrevistados, pues ésta reafirma las elecciones sobre prácticas que pueden resultar nocivas, así como el modo de comprenderlas. La masculinidad es un articulador de las trayectorias de vida de los varones: un hombre trabaja, provee a su familia, bebe, fuma, no asiste al médico, a no ser que sea estrictamente necesario, y no reflexiona sobre qué alimentos consumir, aspectos que están naturalizados o normalizados en el "hacerse hombre". Los anteriores elementos emergen como patrones comunes en las trayectorias de vida de los hombres entrevistados y resultan aspectos relevantes como determinantes de un IAM.

"me la paso recordando el pasado, por ejemplo cuando me emborrachaba; mujer aquí, mujer allí, en cada puerta un amor; fume uno, dos paquetes [de cigarrillo], descaradamente les cuento porque así era mi vida... uno de hombre, eso es así'9-10:9

\section{2. "Mi pequeño modo de entender":} Autocomprensión del fenómeno: Al indagar sobre la manera como se interpreta desde sus marcos de referencia el infarto, resulta interesante la manera como se incorpora el lenguaje biomédico a sus propias construcciones. Una forma de sincretismo que les posibilita a los entrevistados una explicación lógica de la enfermedad. Estas son algunas de las descripciones del IAM cómo fenómeno físico desde los participantes en las que podemos identificar ese sincretismo:

\footnotetext{
"uno cree que el corazón no bota gases y sí, porque es una bomba, al botarlos se encuentra con las grasas, con trombos, que tapan la circulación y ahi está el problema"1-4:111

"considero que un infarto se refiere a la circulación de la sangre, hay grasas y se taponean las vias y no van para donde deben ir y pues ahi se da el infarto"7-5:88

"Aunque el infarto es un problema de arterias, lo relacionan con mi diabetes, demasiada azúcar para que el corazón la mueva" 6-7:5
}

Desde la experiencia de los entrevistados el IAM es un evento inesperado que puede llegar a ser mortal, que se presenta súbitamente con una serie de síntomas físicos anormales y al cual no le atribuyen una causa directa. En un primer momento del evento llegan a considerar que no tiene una causa conocida y tampoco tiene relación inmediata con sus hábitos y elecciones de vida. Hasta cuando hay un proceso de familiarización con la información y recomendaciones médicas, los entrevistados aceptan el daño que sus hábitos generaron.
"Yo creí que me iba a morir ahí. Tenía que hacer un esfuerzo terrible para caminar, yo no podía alzar una pata ni la otra; era una cosa aquí (pecho) y en la cintura, en la espalda era una cosa como ahogarme, como los signos de la muerte" 9 - 10:2
"Sentí como mareo una cosa asi bien fea, me llevaron a urgencias y ahi me dijeron que me estaba infartando. Uno no piensa nada, se le viene todo el mundo encima" $8-9: 6$

Algunos de los síntomas relatados por los entrevistados no están dentro de los enunciados de los síntomas clásicos o comunes para el IAM en la literatura médica, aspecto que resulta relevante en términos de la información y educación para los pacientes. Por otro lado, para la mayoría de los entrevistados, la idea de la muerte está presente desde el inicio de los síntomas, causando temor en algunos y resignación en otros si esa hubiese sido la consecuencia.

En general los entrevistados manifiestan que el evento generó un cambio frente a su perspectiva de vida, reconociéndola como una segunda oportunidad y como un hecho que transforma sus hábitos y condiciones de vida, más aún, uno de los entrevistados refirió que el tratamiento tendría un efecto de limpieza en el organismo:

\footnotetext{
"[con el cateterismo] como que siente que le destaparon las venas y uno siente que en las venas como que... es decir, hay más ánimo. Es un cambio, una limpieza que le hacen... sí, cuando hay una limpieza de sangre eso le quitan a uno esa nicotina y como que uno puede dejar de fumar" 1-1:12

"no fue sino caer al hospital y le cogí un odio al alcohol, yo tomaba mucho aguardiente" 8-9:7

"pues como uno no iba al doctor que le diga qué cosas pueden pasar o las consecuencias son estas y estas [respecto de hábitos como fumar y beber alcohol]... Hasta que a uno le pasan estas cosas, entonces, o lo deja o lo deja [cigarrillo]" 1:6-32
}

Si bien es cierto todos los entrevistados refieren una atención oportuna e integral en el momento del evento, narran que el seguimiento médico se ha convertido en una complicación para ellos, dilatando la entrega de medicamentos y la consulta de control con cardiología. Muchos de ellos deben recurrir a comprar medicamentos por su cuenta. 
"si, tenía [una EPS del régimen subsidiado], vine por el control, yo mostré esa EPS aquí y le dicen a uno que no, que no hay contrato que pague la consulta; entonces no he podido tener control" 5-1:9

"hay medicamentos que me toca comprarlos aparte; me toco comprar porque el seguro le da a uno citas por allá de dos meses, cuando ya se muera uno, a dos meses de distancia, me toca comprar la droga para sentir el alivio" 9-10:9

Así el sistema de salud se convierte en una barrera para el proceso de restablecimiento de la salud, después de sobrevivir al infarto. Esta situación en el contexto colombiano llega a ser común, pues durante los últimos años se ha evidenciado un aumento en las quejas de usuarios por la falta de oportunidades en la atención en salud de manera general. A la vez las complicaciones en la atención hacen parte de entender que la enfermedad está ligada a procesos administrativos que pueden ser tan impactantes como el propio evento.

\section{DISCUSIÓN}

La aproximación comprensiva a las trayectorias que estructuran los estilos de vida de varones que han sufrido infarto agudo de miocardio desde sus voces y representaciones nos muestran cómo el mundo del trabajo sobredetermina muchos de los comportamientos y elecciones de la cotidianidad. Al respecto autores como Cockerham proponen una interpretación de los estilos de vida en relación con la salud como una dialéctica entre las posibilidades (estructura) y las decisiones de vida (agencia); así los comportamientos relacionados con los estilos de vida no son pautas individuales sino roles reconocibles, dentro del grupo o clase social de referencia ${ }^{21}$. Es decir son fenómenos socialmente fundamentados y los resultados del presente estudio reflejan esa dialéctica entre agencia y estructura.

En relación con lo anterior y a partir de los resultados del presente estudio, el concepto convencional de factor de riesgo también se pone en tensión, pues se evidencia que más allá de ser una probabilidad como lo define la epidemiología clásica ${ }^{22}$, un riesgo es el resultado de un tejido histórico y cultural, sobre el cual las personas generan diferentes niveles de concienciación y solo hasta que se expresa como una enfermedad que puede ocasionar la muerte, son considerados como hábitos que producen serios daños, como el caso del infarto. Al respecto Beck, concluye que "es la percepción cultural... lo que constituye el riesgo" ${ }^{23}$ dando un rol central al efecto codificador de la cultura sobre el sentido y la representación de lo que es un riesgo ${ }^{24}$, asunto que puede evidenciarse en los resultados obtenidos.
Los hábitos que emergen en la trayectoria en la vida, como el tabaco y el alcohol, fueron comunes en todos los entrevistados y han sido ampliamente documentados en la literatura científica como factores sobre los que se encuentran mayores diferencias entre hombres y mujeres con enfermedad cardiovacular ${ }^{25-31}$, es decir, es un factor que está presente con mayor frecuencia en hombres, razón por la cual, algunos de estos estudios lo ligan a la construcción de lo masculino. Sin embargo, asuntos como la generalización de la masculinidad, la concentración en el discurso biomédico a la hora de investigar y la falta de exploración de lo experiencial del género como aprendizaje del "ser" pasan desapercibos y por lo tanto son poco comprendidos ${ }^{32,33}$.

Uno de los elementos emergentes en los textos de los entrevistados se relaciona con elecciones comportamientos sobre el beber, fumar, incluso no asistir al médico, los cuales se han explicado desde la naturalización de lo que "significa ser un hombre" como un universal histórico ${ }^{33}$, evidenciando la necesidad de profundizar en la indagación sobre el cómo se construyen las masculinidades desde sus espacios y experiencias privadas 14,34, de modo que posibilite repensar las categorías de género y sus manifestaciones en el proceso salud-enfermedad.

Resulta relevante que al aproximarse a un evento que parece tan clínico, como lo es el IAM, se encuentre en estrecha relación con los roles que desempeñan los varones; lo cual convoca a profundizar en la perspectiva de género o en la comprensión de la salud de los hombres más allá del campo de lo sexual/sexualidad y de la salud mental, en los cuales se han concentrado la mayoría de los estudios sobre masculinidad y salud ${ }^{35}$ y principalmente su valor para pensar las necesidades/ intervenciones en los escenarios del cuidado y la atención en salud. Por otro lado, la salud laboral también encontraría un campo de exploración sobre otros modos de entender los riesgos ocupacionales, pues el mundo del trabajo, en el caso de los entrevistados, se extiende más allá de lo operativo y de la jornada laboral.

\section{AGRADECIMIENTOS}

A los hombres que aceptaron compartir sus historias y experiencias. A Jennifer, Laura, Carol y Lizeth, estudiantes de VIII semestre de Enfermería de la Fundación Universitaria de la Salud por el apoyo para la realización de las entrevistas.

Este proyecto fue financiado con recursos de la convocatoria de capital semilla para proyectos de investigación de la Fundación Universitaria de ciencias de la Salud. 


\section{REFERENCIAS}

1. Organización Mundial de la Salud. Enfermedades Cardiovasculares. Nota descriptiva

2. Instituto Nacional de Salud. Enfermedad Cardiovascular: principal causa de muerte en Colombia. boletín Observatorio Nacional de Salud. Boletín $\mathrm{N}^{\circ} 1$. Diciembre 9 del 2013.

3. Ministerio de Salud y Protección Social. Hombres, más propensos en Colombia a morir de infarto. Boletín de Prensa No 072. Marzo del 2014.

4. Fuster V, Kelly B. (Editors). Promoting Cardiovascular Health in the Developing World. A Critical Challenge to Achieve Global Health. National Academies Press (US); 2010.

5. Kelly B, Narula J, Fuster V. Recognizing Global Burden of Cardiovascular Disease and Related Chronic Diseases. Mt Sinai J Med. 2012; 79: 632640. DOI: $10.1002 / \mathrm{msj} .21345$.

6. Lantz P, House J, Mero R, Williams D. Stress, life events, and socioeconomic disparities in health: results from the Americans' Changing Lives Study, J Health Soc Behav. 2005; 46 (3): 274-288.

7. Pearlin LI, Schieman S, Fazio EM, Meersman SC. Stress, health, and the life Course: some conceptual perspectives. J Health Soc Behav. 2005; 46(2): 205219.

8. Eriksson MK, Franks PW, Eliasson M. A 3-year randomized trial of lifestyle intervention for cardiovascular risk reduction in the primary care setting: the Swedish Björknäs study. PLoS One. 2009;4(4):e5195. DOI: 10.1371/journal. pone. 0005195 .

9. Nolte E, McKee M. Measuring the health of nations: Updating an earlier analysis. Health Aff. 2008; 27: 58-71. DOI: 10.1377/hlthaff.27.1.58.

10. Marmot MG, Shipley M, Brunner E, Hemingway $\mathrm{H}$. Relative contribution of early life and adult socioeconomic factors to adult mortality in the Whitehall 11 study. J Epidemiol Community Health. 2001; 55: 301-307.

11. Manor O, Matthews S, Power C. Health selection: the role of inter- and intra-generational mobility on social inequalities in health. Soc Sci Med. 2003; 57(11): 2217-2227.

12. González-Zobl G, Grau M, Muñoz MA, Marti R, Sanz H, Sala J. Estudio REGICOR. Posición socioeconómica e infarto agudo de miocardio. Estudio caso-control de base poblacional. Rev Esp Cardiol. 2010; 63(9): 1045-1053. DOI: 10.1016/ S0300-8932(10)70226-8.

13. Manderbacka, K. Elovainio, M. La complejidad de la asociación entre posición socioeconómica e infarto agudo de miocardio. Rev Esp Cardiol. 2010; 63(9): 1015-1018.

14. Emslie C, Hunt K. Men, masculinities and heart disease: A Systematic Review of the qualitative literature. Current Sociol. 2009; 57(2): 155-191 DOI: $10.1177 / 0011392108099161$.

15. Frassa J. Rupturas y continuidades en el mundo del trabajo. Trayectorias laborales y valoraciones subjetivas en un estudio de caso. Rev Cuestiones de Sociología. 2007(invierno); 4: 243-266.

16. Lera C, Genolet A, Rocha V, Schoenfeld Z, Guerriera L, Bolcatto S. Trayectorias: un concepto de posibilita pensar y trazar otros caminos en la intervención profesional de trabajo social. Rev Cátedra Paralel. 2007; 4: 33-39.

17. Vasilachis-de-Gialdino I. Los fundamentos ontológicos y epistemológicos de la investigación cualitativa. FQS: Forum Qualit Soc Res. 2009; 10(2): 1-17.

18. Morse J. The significance of saturation. Qual Health Res. 1995; 5(2):147-149.

19. Morse J (Ed.), Asuntos críticos en los métodos de investigación cualitativa. Editorial Universidad de Antioquia. 2003:139-157.

20. Calderón C. El papel de la reflexividad en la investigación cualitativa en salud. Rev Cuatrim Humanid. 2011; 18(55): 43-53.

21. Cockerham W. Health Lifestyle Theory and the Convergence of Agency and Structure. J Health Soc Behav. 2005; 46(1): 51-67.

22. Gómez-Rinesi J. Epidemiologia clínica: riesgo. Rev Posgrado Cátedra VI Med. 2001; 13: 5-14.

23. Beck, Ulrich. Retorno a la Teoría de la "Sociedad del Riesgo". Boletín de la A.G.E. 2000; 30: 9-20.

24. Amezcua M, Palacios Ramírez J. Jóvenes, alcohol y riesgo: Una mirada crítica desde las teorías socioculturales. Ind Enfermería 2014; 23(3): 149-152.

25. Prata J, Quelhas-Martins A, Ramos S, RochaGonçalves F, Coelho R. Gender differences in quality of life perception and cardiovascular risk in a community sample. Rev Port Cardiol. 2016; 35(3): 153-160. DOI: 10.1016/j.repc.2015.09.022.

26. Hunt K, Lewars H, Emslie C, Batty G. Decreased risk of death from coronary heart disease amongst men with higher 'femininity' scores: a general population cohort study. Int J Epidemiol. 2007; 36(3): 612-620.

27. Camelo L, Giatti L, Barbosa-Neves J, Lotufo P, Benseñor I, Chor D, et al. Life Course Socioeconomic Position and C-Reactive Protein: Mediating Role of Health-Risk Behaviors and Metabolic Alterations. The Brazilian Longitudinal Study of Adult Health (ELSA-Brasil). PLoS One. 2014; 9(10): e108426. 
DOI: 10.1371 journal.pone. 0108426 .

28. Andrés E, León M, Cordero A, Magallón R, Magán $\mathrm{P}$, Luengo E, et al. Factores de riesgo cardiovascular y estilo de vida asociados a la aparición prematura de infarto agudo de miocardio. Rev Esp Cardiol. 2011; 64(6): 527-529. DOI: 10.1016/j.recesp.2010.09.012

29. Barrett-Connor E. Commentary: Masculinity, femininity and heart disease. Int J Epidemiol. 2007; 36(3): 621-622

30. George J, Rapsomaniki, E, Pujades-Rodriguez M, Dinesh-Shah A, Denaxas S, Herrett E, et al. How does cardiovascular disease first present in women and men? Incidence of 12 cardiovascular diseases in a contemporary cohort of 1.937 .360 people. Circulation. 2015; 132(14): 1320-1328. DOI: 10.1161/CIRCULATIONAHA.114.013797.

31. Tielemans S, Geleijnse J, Menotti A, Boshuizen H, Soedamah Muthu S, Jacobs D, et al. Ten Year Blood Pressure Trajectories, Cardiovascular Mortality, and Life Years Lost in 2 Extinction Cohorts: the Minnesota Business and Professional Men Study and the Zutphen Study. J Am Heart Assoc. 2015; 4(3): e001378. DOI: 10.1161/JAHA.114.001378.

32. Riska, E. Masculinity and Men's Health: Coronary Heart Disease in Medical and Public Discourse. Rowman \& Littlefield Publishers, 1st Edition. 2006. Helsinki, Finland.

33. Courtenay WH. Constructions of masculinity and their influence on men's well-being: a theory of gender and health. Soc Sci Med. 2000; 50(10): 1385-1401.

34. Figueroa-Perea. J. Un apunte sobre varones y masculinidades enajenadas. Conferencia del III congreso internacional sobre estudios de varones y masculinidades. Colegio de México. México, 2013.

35. Sabo D. Comprender la salud de los hombres: un enfoque relacional y sensible al género. Publicaciòn ocasional No. 4. Editorial Organización Panamericana de la Salud. Harvard Center for Population and DEvelopment Studies. 2000. 